Research Paper

\title{
A Retrospective Review of Chemotherapy for Patients with Small Bowel Adenocarcinoma in British Columbia
}

\author{
Donat Duerri; Susan Ellard²; Yongliang Zhai ${ }^{3}$; Marianne Taylor²; Sanjay Rao ${ }^{2 \bowtie}$ \\ 1. Department of Medical Oncology / Hematology, City Hospital Triemli, Zurich, Switzerland. \\ 2. BC Cancer Agency - Centre for the Southern Interior, Kelowna, Canada. \\ 3. University of British Columbia, Vancouver, Canada. \\ $\square$ Corresponding author: Dr. Donat Dürr, Stadtspital Triemli, Birmensdorferstrasse 497, CH-8063 Zürich, Switzerland. Phone: +41 444163480 Fax: +41 44416 \\ 3524 donat.duerr@triemli.zuerich.ch
}

๑ Ivyspring International Publisher. Reproduction is permitted for personal, noncommercial use, provided that the article is in whole, unmodified, and properly cited. See http://ivyspring.com/terms for terms and conditions.

Received: 2016.06.26; Accepted: 2016.09.18; Published: 2016.11.25

\begin{abstract}
Background: Small bowel adenocarcinoma (SBA) is associated with a poor prognosis. It is an uncommon malignancy and therefore difficult to study. Randomized phase III trials are not available to guide best approaches. The Provincial Cancer Registry of the British Columbia Cancer Agency contains long-term data on patients with SBA. The authors analyzed characteristics and treatment outcomes for SBA patients diagnosed between 1990 and 2008.

Material and methods: Charts of 150 patients with a histological diagnosis of SBA were retrospectively analyzed. Epidemiological and treatment data were collected. Disease-free survival (DFS) and overall survival (OS) were estimated by the Kaplan-Meier method.

Results: Baseline characteristics, such as median age at diagnosis (64.5 years), tumor stage (I-II $33 \%$, III-IV $58 \%$, unknown $9 \%$ ), and location (duodenum $48 \%$, jejunum $31 \%$, ileum $21 \%$ ) were consistent with published data. $55 \%$ of patients had a positive family history of cancer. DFS and OS of 29 patients treated with adjuvant chemotherapy were not significantly different to that of 47 patients without $(p=1$ and $p=0.211$, respectively). In the palliative setting patients treated with polychemotherapy (21 patients) had statistically better OS than patients treated with monochemotherapy (12 patients) $(\mathrm{p}=0.0228)$.

Conclusions: Our study suggests a survival benefit for advanced-stage SBA patients treated with poly- versus monochemotherapy. This, however, was a retrospective analysis with several potential confounders. Nevertheless, our study adds to the evidence suggesting that chemotherapy may be beneficial for patients with SBA, at least in the palliative setting.
\end{abstract}

Key words: small bowel, adenocarcinoma, chemotherapy, adjuvant, palliative.

\section{Introduction}

The small intestine is 5 to $7 \mathrm{~m}$ long and represents $75 \%$ of the length of the entire gastrointestinal tract. However, the incidence rate of small intestinal neoplasms is approximately 11-fold lower than that of colorectal cancer [1]. Speculation on the reasons for this discrepancy are ongoing; short exposure time between mucosa and potential carcinogens, dilution of carcinogens by secretions, and absence of bacterial degradation of bile salts in the small intestine are some of the potential explanations [2].
The term "small bowel neoplasm" encompasses several different histological subtypes. Small bowel adenocarcinoma (SBA) (36.9\%) and carcinoid tumors $(37.4 \%)$ are the most commonly observed subtypes [3]. Whereas the incidence rate of carcinoid has increased recently, the incidence rate of SBA has been quite stable [3].

Unfortunately, as with most upper gastrointestinal tract tumors, the prognosis of SBA is poor. The overall survival of patients with metastatic disease is 9 to 11 months $[4,5]$. Due to the rarity of 
SBA, there are no robust data in the literature to guide treatment decisions in these patients. In that light, we retrospectively analyzed epidemiological data, risk factors and outcomes of patients in British Columbia who were diagnosed with SBA between 1990 and 2008 , to determine whether any strong patterns had emerged from two decades of provincial experience.

\section{Material and Methods}

After approval of this project by University of British Columbia (UBC) British Columbia Cancer Agency (BCCA) Research Ethics Board patients with SBA were identified using the Provincial Cancer Registry of BCCA. This database contains data on patients treated at the BCCA's Vancouver, Fraser Valley, Vancouver Island, Southern Interior and Abbotsford centers. Only patients given a histological diagnosis of SBA between 1990 and 2008 were included in our study, to achieve adequate follow-up details for all included patients. Individual patient charts were reviewed and data recorded.

\section{Statistical analysis}

Disease-free survival (DFS) was defined as time elapsed from histological diagnosis until documented relapse of SBA (date of histological diagnosis of recurrence; if histology was not available, date of CT scan suggesting recurrence). Overall survival (OS) was defined as time from histological diagnosis until death (all causes) or until date of chart review. Estimates of the survival function for DFS and OS were obtained using the Kaplan-Meier method. The Kaplan-Meier curves were drawn to compare the survival functions for different patients and disease characteristics. This comparison was made using the log-rank test.

\section{Results}

Using the above described search criteria the Provincial Cancer Registry identified 189 patients. Basic data such as sex, age at diagnosis of SBA, and tumor location were available on 150 patients; data with respect to treatment benefit were available for 147 patients.

\section{Baseline characteristics}

The median age at diagnosis was 64.5 years (range 24.5 to 92 years) (Table 1). Slightly more than half of the patients were male $(58 \%)$. The most common primary tumor site was duodenum $(48 \%)$, followed by jejunum $(31 \%)$ and ileum $(21 \%) .58 \%$ of patients had locally-advanced (stage III) or metastatic (stage IV) disease at diagnosis, whereas 33\% had stage I or II disease at presentation. Stage could not be determined for $9 \%$ of patients.
Table 1. Baseline characteristics of patients with small bowel adenocarcinoma diagnosed between 1990 and 2008 in British Columbia.

\begin{tabular}{ll}
\hline Characteristics $(\mathrm{n}=150)$ & \\
\hline Age of diagnosis & \\
Mean & 64.2 years \\
Median & 64.5 years \\
Range & $24.5-92.0$ years \\
Duodenum (Median) & 65.0 years \\
Jejunum (Median) & 62.2 years \\
Ileum (Median) & 70.0 years \\
Sex & \\
Male & $87(58 \%)$ \\
Female & $63(42 \%)$ \\
Tumor location & \\
Duodenum & $72(48 \%)$ \\
Jejunum & $46(31 \%)$ \\
Ileum & $32(21 \%)$ \\
Differentiation grad & \\
Good & $10(7 \%)$ \\
Moderate & $71(47 \%)$ \\
Poor & $43(29 \%)$ \\
Not further detemined & $26(17 \%)$ \\
Initial tumor stage & \\
I & $8(6 \%)$ \\
II & $41(27 \%)$ \\
III & $32(21 \%)$ \\
IV & $56(37 \%)$ \\
Unknown & $13(9 \%)$ \\
Site of metastasis & \\
(based on the data of 55 of the 56 patients with stage IV at & \\
diagnosis) & \\
Liver & $23 / 55(42 \%)$ \\
Lung & $6 / 55(11 \%)$ \\
Peritoneum (incl. ovaries, omentum) & $25 / 55(45 \%)$ \\
Non regional lymph nodes & $16 / 55(29 \%)$ \\
Others & $3 / 55(5 \%)$ \\
\hline &
\end{tabular}

Histologically, $47 \%$ of patients had moderately-differentiated, while $29 \%$ and $7 \%$ had poorly- or well-differentiated adenocarcinoma (tumor grading was not available for $17 \%$ of patients). Patients with metastatic disease at diagnosis most commonly had peritoneal $(45 \%)$ or hepatic $(42 \%)$ metastases, followed by distant nodal spread (29\%). Some patients had multiple metastatic sites.

\section{Risk factors for developing SBA}

$55 \%$ of 109 patients for whom family history details were recorded had a positive family history of malignancy (Supplementary Material S1). A patient was determined to have a positive family history if a history of malignancy in one or more first-degree relatives (brother, sister, father, mother) was documented in the chart. Where patients had a positive family history, $20 \%$ and $12 \%$ of the relatives had intestinal tumors or breast cancer respectively. Furthermore, Lynch syndrome was diagnosed in 6 of 109 patients based on the Amsterdam II criteria [6]. The diagnosis of Peutz-Jeghers Syndrome was recorded in one case. Almost a third of patients were 
found to have a synchronous $(8 \%)$ or metachronous $(24 \%)$ second malignancy, most often colorectal malignancies.

With respect to aspects of medical history other than malignancy, Crohn's disease, a known risk factor for developing SBA, was identified in 18 patients $(13 \%) .41 \%$ of patients with a primary diagnosis of ileal SBA had a known history of Crohn's disease, but only $1 \%$ of patients with SBA of the duodenum and $10 \%$ of those with SBA of the jejunum had a similar history.

\section{Symptoms at diagnosis}

Almost $90 \%$ of SBA patients reviewed presented with abdominal discomfort, symptoms of obstruction (typically nausea and/or vomiting), or gastrointestinal bleeding (Supplementary Material S2). Abdominal discomfort was the most common symptom $(33 \%)$, followed by obstruction $(27 \%)$ and gastrointestinal bleeding / chronic iron deficiency (26\%). Jaundice was the leading symptom at diagnosis in $2 \%$ of patients. $12 \%$ of patients were either asymptomatic at diagnosis or presented with symptoms other than those described above.

\section{Chemotherapy in the adjuvant setting}

76 patients were treated initially with curative intent. 29 of those underwent adjuvant chemotherapy, 47 had no further treatment. A variety of adjuvant chemotherapy protocols (bolus 5-FU, capecitabine, capecitabine + oxaliplatin (CAPOX), infusional 5-FU + oxaliplatin (FOLFOX), and infusional 5-FU + irinotecan (FOLFIRI)) were used. DFS in the group treated with adjuvant chemotherapy was not significantly different from the group not treated with adjuvant chemotherapy (Supplementary Material S3). The same was true for the overall survival of both groups (HR 1.12, $p=0.211$ ) (Figure 1).

\section{Chemotherapy in the palliative setting}

In the palliative setting, however, patients treated with chemotherapy had a statistically significant improvement in OS compared to patients not treated with chemotherapy ( $p=0.00012$, Figure 2). Chemotherapy protocols included cisplatin plus 5-FU, gemcitabine, bolus 5-FU, continuous infusional 5-FU, capecitabine, FOLFIRI, FOLFOX, CAPOX, and capecitabine plus irinotecan (CAPIRI). Of the 33 patients treated with palliative chemotherapy, 21 had a chemotherapy regimen containing at least two agents. Overall survival of those patients was significantly longer than that of patients treated with single agent chemotherapy $(p=0.0228$, Figure 3$)$.

\section{Discussion}

The primary purpose of this review was to obtain more current data about SBA with regard to epidemiology, risk factors, prognosis and chemotherapy efficacy. The records of 150 patients diagnosed with SBA between 1990 and 2008 in British Columba, Canada were individually reviewed.

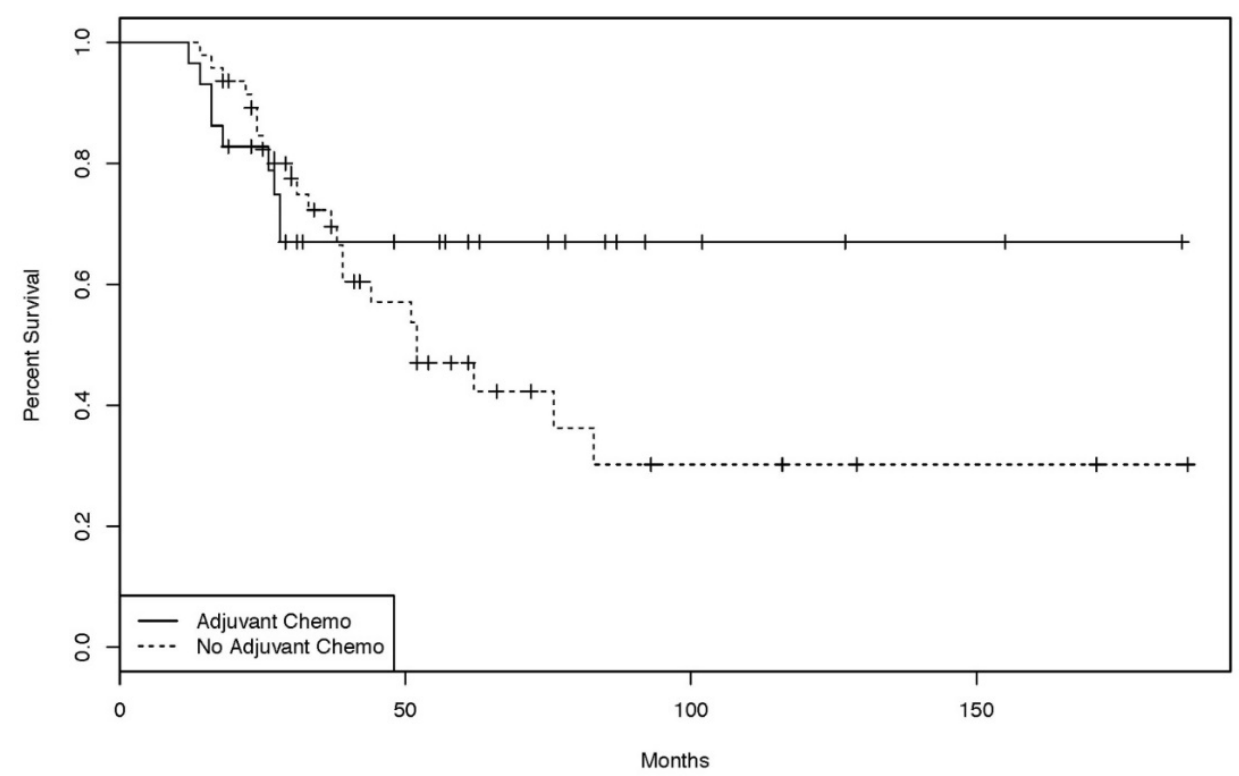

Figure 1. Kaplan-Meier curves of the overall-survival for patients treated with or without adjuvant chemotherapy. [47 patients treated in curative intent without adjuvant chemotherapy, 29 patients treated in a curative intent with adjuvant chemo- or chemoradiotherapy. (log rank test: $p=0.211)]$. 


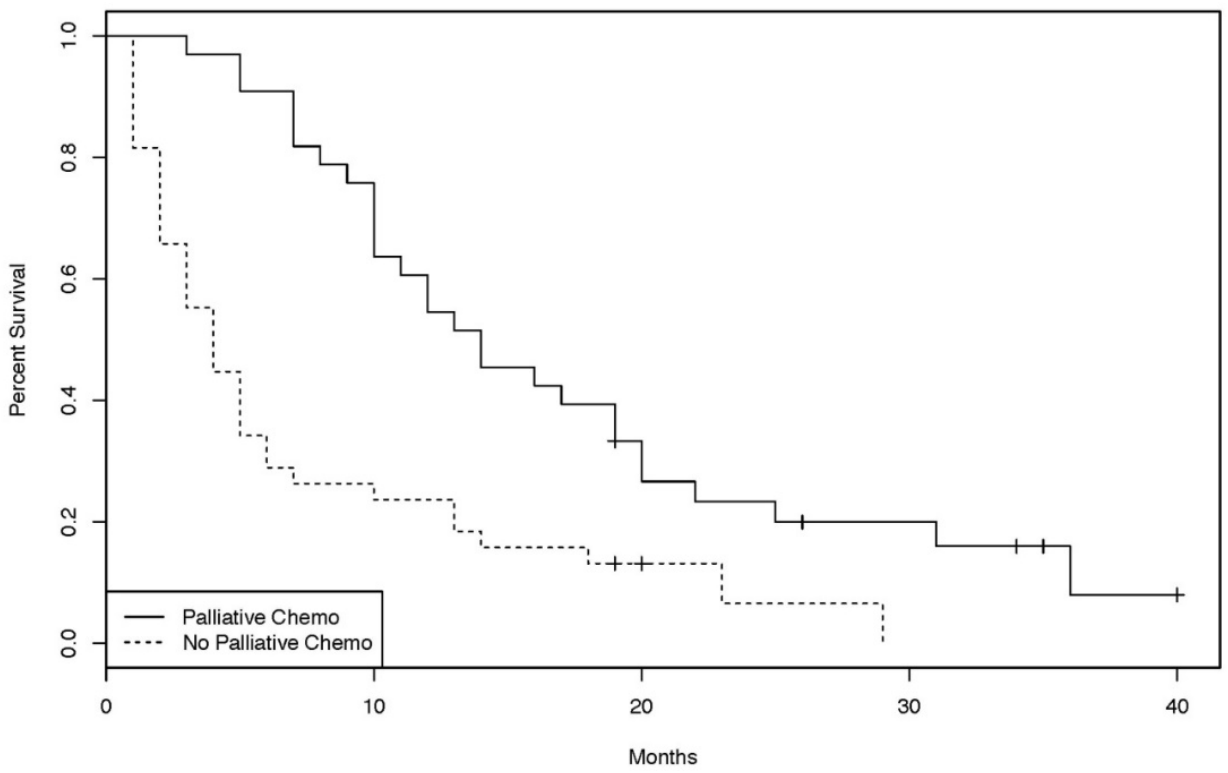

Figure 2. Kaplan-Meier curves of the overall survival for patients treated with or without palliative chemotherapy. [ 38 patients without palliative chemotherapy, 33 patients with palliative chemotherapy. (log-rank test: $p=0.00012)]$

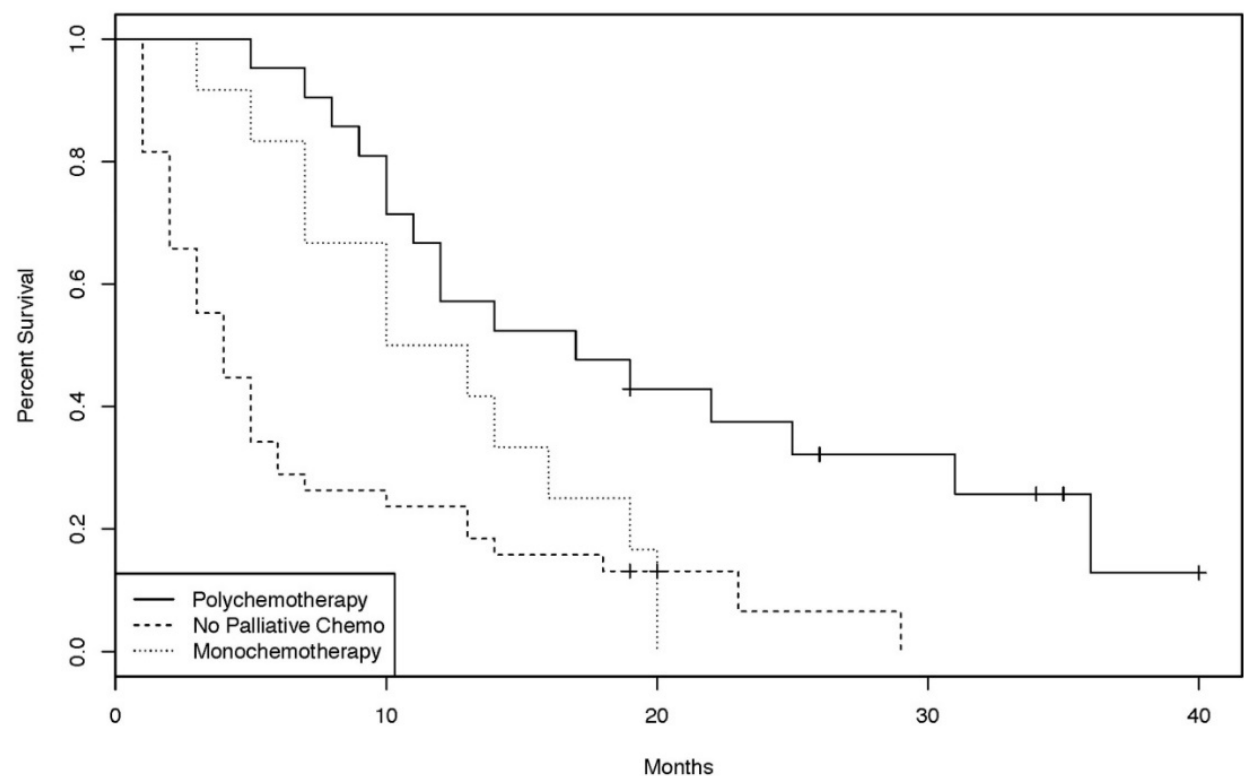

Figure 3. Overall survival Kaplan-Meier curves of patients treated with monochemotherapy, polychemotherapy, or no palliative chemotherapy. [38 patients without palliative chemotherapy, 21 with polychemotherapy and 12 with monochemotherapy. (log-rank test: $p=0.0228)]$

There are many epidemiological studies on patients with SBA in the published literature $[3,4,5$ among others]. Though our current study involved relatively few patients, the data were consistent with published literature. For example, the current study identified a slight male predominance $(58 \%$ in our study versus $54 \%$ in the literature), similar median age at diagnosis (64.5 versus 67 years) and similar distribution of primary tumor site of origin. In about half of patients, the primary tumor was located in the duodenum ( $48 \%$ versus $56 \%$ in the literature [3]), followed by jejunum (31\%) and ileum (21\%). With respect to prognosis our data were also very consistent with the literature; the median survival of 22 months compares favorably to the median survival of approximately 20 months described in other published sources [4].

The pathogenesis of SBA is not yet fully understood. The literature describes genetic factors (such as familial adenomatous polyposis (FAP), hereditary non-polyposis colorectal cancer (HNPCC), Peutz-Jeghers Syndrome); environmental factors (such as tobacco, alcohol use, and a diet high in red meat); and predisposing medical conditions (such as Crohn's or celiac disease) [7]. 55\% of our patients had a first-degree relative with a history of cancer and 
Lynch Syndrome (Amsterdam II criteria) and Peutz-Jeghers Syndrome were identified in 6\% and $1 \%$ respectively. These data support the hypothesis that genetic predisposition in general and Lynch syndrome in particular may play an important role in the development of SBA [8, 9]. Crohn disease as a known predisposing medical condition was identified in $13 \%$ of our patients, with the highest proportion in the group of patients with ileal-primary SBA (41\%). $72 \%$ of patients with Crohn's disease who developed SBA were found to have an ileal primary, consistent with findings in other published literature [10].

The management of patients with stage II and III disease remains challenging. Despite surgery with curative intent, the 3-year survival rate for patients with stage III SBA is only 45\% [4]. Adjuvant chemotherapy, radiation or chemo-radiotherapy may be reasonable approaches to reducing recurrence risk. However, due to the rarity of this disease, there are no randomized, controlled, prospective trial data supporting any particular adjuvant strategy [11]. All studies dealing with this question are retrospective analyses and - with the exception of one recent large study [12] - none demonstrated an overall survival benefit for patients treated with adjuvant chemotherapy [5, 13-16]. Consistent with most of the published literature, the 29 patients treated with adjuvant chemotherapy in the present study did not have a statistically significant outcome improvement (either DFS or OS) over the 47 patients who did not receive further treatment (Supplementary Material S3 and Figure 1); however, there was a trend towards benefit (Figure 1).

The retrospective nature of this analysis implies that putative prognostic factors such as tumor stage, residual tumor, lymph node metastasis and vascular invasion [16-19] might not be well balanced; this imbalance might explain, in part, the lack of a statistically significant benefit. The recipients of adjuvant chemotherapy in the present study had more advanced disease at presentation (stage IIIB 23\% versus $4 \%)$, with a minority in stage I $(15 \%$ versus $0 \%$. Despite the discrepancy in prognostic parameters the overall survival of the adjuvant chemotherapy group was not worse, suggesting adjuvant chemotherapy might compensate to some degree for negative prognostic factors. Admittedly, however, the value of this analysis is fairly limited. The simple size in our study was likely inadequate to demonstrate the value of adjuvant chemotherapy. Ideally, a large multinational, randomized, controlled trial could be initiated to determine the value of adjuvant chemotherapy.

In the palliative setting however, there is perhaps more evidence to suggest a benefit for chemotherapy (Figure 2). 33 patients underwent palliative chemotherapy and had a statistically significantly improvement in overall survival compared to untreated patients. Again, prognostic factors might not be well balanced between the two groups, but now favoring the chemotherapy group, who may have been more fit, or had lower disease burden, compared to the group which did not receive chemotherapy. According to the literature, ECOG performance status (0-1 versus 2) is one of the strongest prognostic factors in patients with SBA [16, 20]. Performance status scores could not be reliably obtained for patients in the present study; therefore, it is possible that apparent OS benefit is explained by uneven distribution of performance status, in addition to other potential patient or histological confounders. Nonetheless, the present study lends support to the hypothesis that palliative chemotherapy may benefit patients with advanced SBA.

If chemotherapy has a positive impact on outcomes in patients with incurable metastatic SBA, then which regimen is the most beneficial? Several phase II studies examining a variety of protocols with different OS have been published [21-25]. There are also retrospective single centre experiences comparing different regimens $[5,15,16,20,26-30]$. Current expert opinion favors use of a fluoropyrimidine-platinum combination in the first line $[20,26,27]$. However, definitive data for treatment selection are lacking.

In British Columbia, the variety of palliative regimens employed (FOLFIRI, FOLFOX, CAPOX, cisplatin plus infusional 5-fluorouracil, carboplatin plus docetaxel, single-agent bolus or continuous infusion 5-fluorouracil, capecitabine, gemcitabine) reflects the lack of a consensus approach to systemic therapy and precluded any meaningful subgroup analyses. A simple comparison was undertaken between the outcome of patients receiving combination chemotherapy (FOLFIRI, FOLFOX, CAPOX, cisplatin plus infusional 5-fluorouracil, carboplatin plus docetaxel) and those receiving single-agent chemotherapy (bolus or continuous infusion 5-fluorouracil, capecitabine, gemcitabine). Interestingly, there was a statistically significant overall survival benefit for the combination group (Figure 3). However, it should be recognized that this is a subgroup analysis within a small retrospective study with several potential confounders; definitive statements on the benefit of combination versus single-agent therapy are not possible. Also unknown is the benefit of addition of targeted therapies (for example, bevacizumab and cetuximab) to standard palliative cytotoxic chemotherapy, as these agents are not approved for use in patients with SBA in British 


\section{Columbia.}

In summary, this retrospective analysis of 150 patients with SBA represents a further contribution to the literature on this uncommon entity. Of particular interest are the findings concerning the role of chemotherapy in the management of patients with SBA. In the adjuvant setting, chemotherapy could potentially compensate for poor prognostic factors; however there was not a statistically significant benefit. In the palliative setting, patients treated with chemotherapy appeared to have a significantly longer OS than untreated patients. The data also suggest that combination chemotherapy is more effective than single-agent chemotherapy in the palliative setting. The high frequency of positive family history for other adenocarcinoma as well as the association of this rare condition with one of a small number of genetic or heritable syndromes raise hope that, in this era of molecular discovery, targeted therapy approaches may offer a way forward. One promising approach might be PD1-blockade in patients whose tumors demonstrate mismatch-repair deficiency [31]. Significant limitations of this study are its retrospective nature, lack of consistently available data across all data fields, and modest numbers. The results are best interpreted in the context of the body of literature on SBA as a whole, and may be useful in further hypothesis generation.

\section{Supplementary Material}

S1-S3. http://www.jcancer.org/v07p2290s1.pdf

\section{Conflict of Interest}

The authors have declared no conflicts of interest and no financial disclosures.

\section{References}

1. Siegel R, Ma J, Zou Z, Jemal A. Cancer statistics, 2014. CA Cancer J Clin 2014; 64: 9-29.

2. Gill SS, Heuman DM, Mihas AA. Small intestinal neoplasms. J Clin Gastroenterol 2001; 33: 267-82.

3. Bilimoria KY, Bentrem DJ, Wayne JD, Ko CY, Bennett CL, Talamonti MS. Small bowel cancer in the United States: changes in epidemiology, treatment, and survival over the last 20 years. Ann Surg 2009; 249: 63-71.

4. Howe JR, Karnell LH, Menck HR, Scott-Conner C. The American College of Surgeons Commission on Cancer and the American Cancer Society. Adenocarcinoma of the small bowel: review of the National Cancer Data Base, 1985-1995. Cancer 1999; 86: 2693-706.

5. Halfdanarson TR, McWilliams RR, Donohue JH, Quevedo JF. A single-institution experience with 491 cases of small bowel adenocarcinoma. Am J Surg 2010; 199: 797-803.

6. Vasen HF, Watson P, Mecklin JP, Lynch HT. New clinical criteria for hereditary nonpolyposis colorectal cancer (HNPCC, Lynch syndrome) proposed by the International Collaborative group on HNPCC. Gastroenterology 1999; 116: 1453-6.

7. Zouhairi ME, Venner A, Charabaty A, Pishvaian MJ. Small bowel adenocarcinoma. Curr Treat Options Oncol 2008; 9: 388-99.

8. Delaunoit T, Neczyporenko F, Limburg PJ, Erlichman C. Pathogenesis and risk factors of small bowel a adenocarcinoma: a colorectal cancer sibling? Am J Gastroenterol 2005; 100: 703-10.

9. Park JG, Kim DW, Hong CW et al. Germ line mutations of mismatch repair genes in hereditary nonpolyposis colorectal cancer patients with small bowel cancer: International Society for Gastrointestinal Hereditary Tumours Collaborative Study. Clin Cancer Res 2006; 12: 3389-93.
10. Feldstein RC, Sood S, Katz S. Small bowel adenocarcinoma in Crohn's disease. Inflamm Bowel Dis 2008; 14: 1154-7.

11. Singhal N, Singhal D. Adjuvant chemotherapy for small intestine adenocarcinoma. Cochrane Database Syst Rev 2007 Jul 18;(3):CD005202.

12. Ecker BL, McMillan MT, Datta J et al. Efficacy of adjuvant chemotherapy for small bowel adenocarcinoma: A propensity score-matched analysis. Cancer 2016; 122: 693-701.

13. Overman MJ, Kopetz S, Lin E, Abbruzzese JL, Wolff RA. Is there a role for adjuvant therapy in resected adenocarcinoma of the small intestine. Acta Oncol 2010; 49: 474-9.

14. Koo DH, Yun SC, Hong YS et al. Adjuvant chemotherapy for small bowel adenocarcinoma after curative surgery. Oncology 2011; 80: 208-13.

15. Dabaja BS, Suki D, Pro B, Bonnen M, Ajani J. Adenocarcinoma of the small bowel: presentation, prognostic factors, and outcome of 217 patients. Cancer 2004; 101: 518-26.

16. Fishman PN, Pond GR, Moore MJ et al. Natural history and chemotherapy effectiveness for advanced adenocarcinoma of the small bowel: a retrospective review of 113 cases. Am J Clin Oncol 2006; 29: 225-31.

17. Khan K, Peckitt C, Sclafani F et al. Prognostic factors and treatment outcomes in patients with Small Bowel Adenocarcinoma (SBA): The Royal Marsden Hospital (RMH) experience. BMC Cancer. 2015 Jan 21;15:15.

18. Overman MJ, Hu CY, Wolff RA, Chang GJ. Prognostic value of lymph node evaluation in small bowel adenocarcinoma: analysis of the surveillance, epidemiology, and end results database. Cancer 2010; 116: 5374-82.

19. Chang HK, Yu E, Kim J et al. Adenocarcinoma of the small intestine: a multi-institutional study of 197 surgically resected cases. Hum Pathol 2010; 41: 1087-96.

20. Zaanan A, Costes L, Gauthier M et al. Chemotherapy of advanced small-bowel adenocarcinoma: a multicenter AGEO study. Ann Oncol 2010; 21: 1786-93.

21. Overman MJ, Varadhachary GR, Kopetz $\mathrm{S}$ et al. Phase II study of capecitabine and oxaliplatin for advanced adenocarcinoma of the small bowel and ampulla of Vater. J Clin Oncol 2009; 27: 2598-603.

22. Xiang XJ, Liu YW, Zhang L et al. A phase II study of modified FOLFOX as first-line chemotherapy in advanced small bowel adenocarcinoma. Anticancer Drugs 2012; 23: 561-6.

23. Gibson MK, Holcroft CA, Kvols LK, Haller D. Phase II study of 5-fluorouracil, doxorubicin, and mitomycin $\mathrm{C}$ for metastatic small bowel adenocarcinoma. Oncologist 2005; 10: 132-7.

24. Ono M, Shirao K, Takashima A et al. Combination chemotherapy with cisplatin and irinotecan in patients with adenocarcinoma of the small intestine. Gastric Cancer 2008; 11: 201-5.

25. Crawley C, Ross P, Norman A, Hill A, Cunningham D. The Royal Marsden experience of a small bowel adenocarcinoma treated with protracted venous infusion 5-fluorouracil. Br J Cancer 1998; 78: 508-10.

26. Tsushima T, Taguri M, Honma $\mathrm{Y}$ et al. Multicenter retrospective study of 132 patients with unresectable small bowel adenocarcinoma treated with chemotherapy. Oncologist 2012; 17: 1163-70.

27. Overman MJ, Kopetz S, Wen S et al. Chemotherapy with 5-fluorouracil and a platinum compound improves outcomes in metastatic small bowel adenocarcinoma. Cancer 2008; 113: 2038-45.

28. Czaykowski P, Hui D. Chemotherapy in small bowel adenocarcinoma: 10-year experience of the British Columbia Cancer Agency. Clin Oncol (R Coll Radiol) 2007; 19: 143-9.

29. Koo DH, Yun SC, Hong YS et al. Systemic chemotherapy for treatment of advanced small bowel adenocarcinoma with prognostic factor analysis: retrospective study. BMC Cancer 2011; 11: 205

30. Locher C, Malka D, Boige V et al. Combination chemotherapy in advanced small bowel adenocarcinoma. Oncology 2005; 69: 290-4.

31. Le DT, Uram JN, Wang $\mathrm{H}$ et al. PD-1 Blockade in tumors with mismatch-repair deficiency. New Engl J Med 2015; 372: 2509-20. 